Series A

I. MATHEMATICA

510

\title{
ISOMORPHISMS OF SPACES AND CONVOLUTION ALGEBRAS OF FUNCTIONS
}

BY

KARI YLINEN

H E L S I N K I 972

S U O M A L A N EN TIEDEAKATEMIA

doi:10.5186/aasfm.1972.510 
Copyright (c) 1972 by

Academia Scientiarum Fennica

ISBN $951-41-0037-9$

Communicated 11 February 1972 by Lauri Mrrbert 


\section{Introduction}

Let $G_{1}$ and $G_{2}$ be locally compact Hausdorff topological groups. In this paper we characterize the isometric and bipositive isomorphisms between the convolution algebras $C_{c}\left(G_{j}\right)$ of real or complex valued continuous functions with compact support. It turns out that such isomorphisms are precisely those induced by a topological isomorphism between the groups, followed by multiplication with a continuous multiplicative function which is positive in the bipositive and has modulus one in the isometric case (Theorem 4.1). Our method of proof is based on a characterization of the bipositive and isometric linear isomorphisms between the spaces $C_{c}\left(S_{j}\right)$ for two locally compact Hausdorff spaces $S_{1}$ and $S_{2}$ (Theorem 3.1). Theorem 4.1 is a sharpening of a result of R. E. Edwards ([2] Theorem 2) who showed that the existence of either type of isomorphism implies that the groups $G_{j}$ are topologically isomorphic.

In the case of two compact or locally finite discrete groups $G_{j}$ with normalized Haar measure the general form of a convolution algebra isomorphism $T: C_{c}\left(G_{1}\right) \rightarrow C_{c}\left(G_{2}\right)$ has been determined by $\mathrm{G}$. V. Wood [7] under the hypothesis that $T$ is merely norm-decreasing. However, in our more general situation this assumption is not even sufficient to guarantee that the underlying groups are algebraically isomorphic. Indeed, there are two non-isomorphic finite groups $G_{j}$ admitting a (by finitedimensionality topological) convolution algebra isomorphism $T$ : $C_{c}\left(G_{1}\right) \rightarrow C_{c}\left(G_{2}\right) \quad$ (see [6] p. 306), and as Wood remarks in [7], p. 775, the Haar measure of $G_{2}$ may be so adjusted that $T$ divided by its norm is a norm-decreasing isomorphism.

\section{Notation}

For a locally compact Hausdorff space $S$, let $C_{c}(S, \mathbf{R})$ and $C_{c}(S, \mathbf{C})$ (resp. $C_{0}(S, \mathbf{R})$ and $\left.C_{0}(S, \mathbf{C})\right)$ denote the spaces of the continuous real and complex valued functions on $S$ having compact support (resp. vanishing at infinity). The spaces are regarded as equipped with the uniform norm: $\|f\|=\sup _{x \in S}|f(x)|$. The letter $\mathbf{K}$ is used to refer (consistently) to 
both the real field $\mathbf{R}$ and the complex field $\mathbf{C}$. We often write for short $C_{0}(S), C_{c}(S)$ instead of $C_{0}(S, \mathbf{K}), C_{c}(S, \mathbf{K})$. For a function $f$ on $S$ we write $f \geq 0$ if $f$ is real valued and $f(x) \geq 0$ for all $x \in S$. An operator $T: C_{c}\left(S_{1}\right) \rightarrow C_{c}\left(S_{2}\right)$ or $T: C_{0}\left(S_{1}\right) \rightarrow C_{0}\left(S_{2}\right)$ is bipositive if $T f \geq 0$ is equivalent to $f \geq 0$.

\section{Isomorphisms of function spaces}

In the isometric case the proof of the next theorem is essentially one of the standard proofs of the well-known Banach-Stone theorem, usually stated for compact spaces (see e.g. [5] pp. 334-335). For classical results in the bipositive case see [3] and [4].

Theorem 3.1. Let $S_{1}$ and $S_{2}$ be locally compact Hausdorff spaces and $T: C_{0}\left(S_{1}\right) \rightarrow C_{0}\left(S_{2}\right)$ a vector space isomorphism.

(i) $T$ is isometric if and only if there exist a homeomorphism $\alpha: S_{2} \rightarrow S_{1}$ and a continuous $\mathbf{K}$-valued function $h$ on $S_{2}$ such that $|h(y)|=1$ for all $y \in S_{2}$ and

$$
T f(y)=h(y) f(\alpha(y)), f \in C_{0}\left(S_{1}\right), y \in S_{2} .
$$

(ii) $T$ is bipositive if and only if there exist a homeomorphism $\alpha: S_{2} \rightarrow S_{1}$ and a continuous everywhere positive function $h$ on $S_{2}$ for which the formula (1) above holds.

The theorem remains valid if $C_{0}\left(S_{j}\right)$ is replaced throughout by $C_{c}\left(S_{j}\right)$, $j=1,2$.

Proof. In each case the sufficiency of the existence of $\alpha$ and $h$ with the stated properties is obvious. To begin the proof of necessity let us observe that, as a consequence of Urysohn's lemma, a set $G \subset S_{j}$ is open if and only if for any $x \in G$ there is a function $f$ in $C_{0}\left(S_{j}\right)$ (resp. in $C_{c}\left(S_{j}\right)$ ) with $f(x) \neq 0$ and $f(y)=0$ for all $y \in S_{j} \backslash G$. It follows that if $T$ : $C_{0}\left(S_{1}\right) \rightarrow C_{0}\left(S_{2}\right) \quad$ (resp. $T: C_{c}\left(S_{1}\right) \rightarrow C_{c}\left(S_{2}\right)$ ) and $\alpha: S_{2} \rightarrow S_{1}$ are bijections and $h: S_{2} \rightarrow \mathbf{K}$ a nowhere vanishing function such that formula (1) holds, then $\alpha$ and $\alpha^{-1}$ map open sets onto open sets, i.e. $\alpha$ is a homeomorphism. Therefore $h$, too, is continuous, being locally the quotient of two continuous functions. We are left with the task of constructing $\alpha$ and $h$.

Let us first consider the case of a bipositive isomorphism $T: C_{0}\left(S_{1}, \mathbf{C}\right)$ $\rightarrow C_{0}\left(S_{2}, \mathbf{C}\right)$. The non-zero multiplicative linear forms on the commutative $C^{*}$-algebra $C_{0}\left(S_{j}, \mathbf{C}\right)$ are precisely the pure states of $C_{0}\left(S_{j}, \mathbf{C}\right.$ ) (see [1], 2.5.2). Let $P_{j}$ denote the convex cone of the positive linear forms on $C_{0}\left(S_{j}, \mathbf{C}\right)$. It follows easily from the definition ([1], 2.5.2) that a non-zero functional $\varphi \in P_{j}$ belongs to the ray $\{\lambda \gamma \mid \lambda \in \mathbf{R}, \lambda>0\}$ generated by some pure state $\gamma$ if and only if $\varphi-\psi \in P_{j}$ for $\psi \in P_{j}$ implies that 
$\psi=\mu \varphi$ for some real number $\mu, 0 \leq \mu \leq 1$. This property is obviously preserved by the algebraic transpose $T^{*}$ of $T$ since it maps $P_{2}$ bijectively onto $P_{1}$. In particular, for each pure state $\gamma$ of $C_{0}\left(S_{2}, \mathbf{C}\right), T^{*} \gamma$ is a positive multiple of a pure state of $C_{0}\left(S_{1}, \mathbf{C}\right)$. Therefore, if we identify $S_{j}$ via the natural evaluation map with the set of the non-zero multiplicative linear functionals on $C_{0}\left(S_{j}, \mathbf{C}\right)$, we get a bijection $\alpha: S_{2} \rightarrow S_{1}$ by dividing $T^{*} y$ with a positive number $h(y)$ for each $y \in S_{2}$, i.e.

$$
f(\alpha(y))=\left\langle f, \frac{1}{h(y)} T^{*} y\right\rangle=\frac{1}{h(y)} T f(y), f \in C_{0}\left(S_{1}, \mathbf{C}\right) .
$$

The case of a bipositive isomorphism $T: C_{0}\left(S_{1}, \mathbf{R}\right) \rightarrow C_{0}\left(S_{2}, \mathbf{R}\right)$ is reduced to the above by considering $C_{0}\left(S_{j}, \mathbf{R}\right)$ as a real linear subspace of $C_{0}\left(S_{j}, \mathbf{C}\right)$ and extending $T$ to an operator $T_{c}$ from $C_{0}\left(S_{1}, \mathbf{C}\right)$ to $C_{0}\left(S_{2}, \mathbf{C}\right)$ by setting $T_{c}(f+i g)=T f+i T g$.

Suppose next that $T: C_{0}\left(S_{1}, \mathbf{K}\right) \rightarrow C_{0}\left(S_{2}, \mathbf{K}\right)$ is an isometric isomorphism. We make use of the well-known fact that the extreme points of the unit ball $U_{j}$ of the Banach space adjoint of $C_{0}\left(S_{j}, \mathbf{R}\right)$ (resp. $C_{0}\left(S_{j}, \mathbf{C}\right)$ ) are precisely the Dirac measures multiplied by a real (resp. complex) number with modulus one. As the transpose of $T$ maps the extreme points of $U_{2}$ onto the extreme points of $U_{1}$, and the Dirac measures on $S_{j}$ may be identified with the points of $S_{j}$, we obtain as above a bijection $\alpha: S_{2} \rightarrow S_{1}$ and a function $h: S_{\mathbf{2}} \rightarrow \mathbf{K}$ with $|h(y)|=1$ for all $y \in S_{2}$, such that (1) holds.

Since $C_{c}\left(S_{j}\right)$ is norm dense in $C_{0}\left(S_{j}\right)$, the assertion concerning a linear isometry from $C_{c}\left(S_{1}\right)$ onto $C_{c}\left(S_{2}\right)$ may be proved by reducing it to the previous case.

Finally, let us assume that $T: C_{c}\left(S_{1}, \mathbf{K}\right) \rightarrow C_{c}\left(S_{2}, \mathbf{K}\right)$ is a bipositive isomorphism. Then a separate argument is needed (see the remark below).

We denote $C_{c}\left(S_{j}\right)^{+}=\left\{f \in C_{c}\left(S_{j}, \mathbf{R}\right) \mid f(x) \geq 0\right.$ for all $\left.x \in S_{j}\right\}$,

and

$$
P_{f}=\left\{x \in S_{j} \mid f(x)>0\right\} \text { for } f \in C_{c}\left(S_{j}\right),
$$

$$
F_{x}=\left\{f \in C_{c}\left(S_{j}\right)^{+} \mid f(x)>0\right\} \quad \text { for } x \in S_{j}, \quad j=1,2 .
$$

The closure of $P_{f}$ for $f \in C_{c}\left(S_{j}\right)^{+}$is denoted $\operatorname{supp}(f)$ as usual. Let us first show that

$$
\bigcap_{f \in F_{x}} \operatorname{supp}(T f) \neq \varnothing, x \in S_{1}
$$

As every $\operatorname{supp}(T f)$ is compact, it is enough to prove that $\bigcap_{f \in F} \operatorname{supp}(T f) \neq \varnothing$ for every finite subset $F$ of $F_{\boldsymbol{x}}$. There exist a constant $\delta>0$, a neighbourhood $U$ of $x$ such that $f(t)>\delta$ on $U$ for each $f \in F$, and a func- 
tion $g \in C_{c}\left(S_{1}\right)^{+}$with maximum value $\delta$ and support contained in $U$. The positivity of $T$ then implies $\bigcap_{f \in F} \operatorname{supp}(T f) \supset \operatorname{supp}(T g) \neq \varnothing$. Next we make use of (2) in proving the stronger statement

$$
A_{x}=\bigcap_{f \in F_{\boldsymbol{x}}} P_{T f} \neq \varnothing, x \in S_{1} .
$$

Indeed, by (2) it is sufficient to find for each $f \in F_{x}$ a function $g \in F_{x}$ such that $\operatorname{supp}(T g) \subset P_{T f}$. The function $g$ may be constructed as follows. Choose $f_{1} \in C_{c}\left(S_{1}\right)^{+}$and $f_{2} \in C_{c}\left(S_{2}\right)^{+}$so that $f_{1}(x)>0$ and $f_{2}$ is positive on $\operatorname{supp}(T f)$. When the function $f_{1}+T^{-1} f_{2}$ is multiplied with a small enough positive number, we ohtain a function $f_{3} \in C_{c}\left(S_{1}\right)^{+}$such that $0<f_{3}(x)<f(x)$ and $T f_{3}$ is greater than a positive constant on $\operatorname{supp}(T f)$. Now define $g=f-\inf \left(f, f_{3}\right)$, so that by the linearity and bipositivity of $T$ we have $T g=T f-\inf \left(T f, T f_{3}\right)$. Then

$$
\operatorname{supp}(T g) \subset\left\{y \in S_{2} \mid T f(y) \geq \inf _{t \in \operatorname{supp}(T f)} T f_{3}(t)\right\} \subset P_{T f},
$$

since the middle set is closed and in its complement $T g(y)=T f(y)-$ $T f(y)=0$. Clearly, $g \in C_{c}\left(S_{1}\right)^{+}$and $g(x)>0$. Thus $g$ has all the desired properties, and (3) follows. Suppose now $y \in A_{x}$. We show that $F_{x}=T^{-1}\left(F_{y}\right)$. First of all, it is clear that $F_{x} \subset T^{-1}\left(F_{y}\right)$. Using this after applying (3) to $T^{-1}$ and $y$, and then observing that $\bigcap_{f \in F_{x}} P_{f}=\{x\}$ we have, conversely, for any $g \in T^{-1}\left(F_{y}\right)$

$$
\varnothing \neq \bigcap_{f \in T^{-1}\left(F_{y}\right)} P_{f} \subset P_{g} \cap\left(\bigcap_{f \in F_{x}} P_{f}\right) \subset\{x\} .
$$

i.e. $x \in P_{g}$ or $g \in F_{x}$. Thus the sets $F_{x}$ and $F_{y}$ are by symmetry in one-to-one correspondence via $T$, and as $F_{x}$ determines $x$ uniquely, we get a bijection $\alpha: S_{2} \rightarrow S_{1}$ with the property $T^{-1}\left(F_{y}\right)=F_{\alpha(y)}, y \in S_{2}$, which implies

$$
P_{T f}=\alpha^{-1}\left(P_{f}\right), f \in C_{c}\left(S_{1}\right)^{-} .
$$

The function $h$ is now defined by choosing for each $y \in S_{2}$ a function $f \in C_{c}\left(S_{1}\right)^{+}$with $f(\alpha(y))=1$ and setting $h(y)=T f(y)$. It follows from (4) that $h(y)>0$ and this definition is independent of the choice of $f$, for if $f_{1}(\alpha(y))=f_{2}(\alpha(y))=1$, the non-negative functions $f_{1}-f^{\prime}$ and $f_{2}-f^{\prime}$, where $f^{\prime}=\inf \left(f_{1}, f_{2}\right)$, vanish at $\alpha(y)$, so $T f_{1}-T f^{\prime}$ and $T f_{2}-T f^{\prime}$ vanish at $y$, i.e. $T f_{1}(y)=T f_{2}(y)$. Thus, in particular,

$$
h(y) f(\alpha(y))=T\left(\frac{1}{f(\alpha(y))} f\right)(y) f(\alpha(y))=T f(y)
$$

for any $f \in C_{c}\left(S_{1}\right)^{+}$with $f(\alpha(y))>0$. If $f \in C_{c}\left(S_{1}\right)^{+}$and $f(\alpha(y))=0$, 
the equation $T f(y)=h(y) f(\alpha(y))$ is also given by (4). As every $f \in C_{c}\left(S_{1}\right)$ may be expressed as a linear combination of some elements of $C_{c}\left(S_{1}\right)^{+}$, the proof of this last part of the theorem is also complete.

Remark. Unlike the isometric case, the proof of the above result involving a bipositive isomorphism $T: C_{c}\left(S_{1}\right) \rightarrow C_{c}\left(S_{2}\right)$ cannot be reduced to the part of the theorem concerned with the spaces $C_{0}\left(S_{j}\right)$, since a bipositive isomorphism $T: C_{c}\left(S_{1}\right) \rightarrow C_{c}\left(S_{2}\right)$ is not always the restriction to $C_{c}\left(S_{1}\right)$ of any bipositive isomorphism from $C_{0}\left(S_{1}\right)$ onto $C_{0}\left(S_{2}\right)$. For example, it is quickly seen from the above theorem that any bipositive isomorphism $T_{\mathbf{0}}: C_{\mathbf{0}}(\mathbf{R}, \mathbf{R}) \rightarrow C_{0}(\mathbf{R}, \mathbf{R})$ extending the mapping $T: C_{c}(\mathbf{R}, \mathbf{R}) \rightarrow C_{c}(\mathbf{R}, \mathbf{R}), T f(x)=\left(1+x^{2}\right) f(x)$, should also have the form $T_{0} f(x)=\left(1+x^{2}\right) f(x)$, which is impossible, as e.g. the function $x \mapsto\left(1+x^{2}\right)\left(1+x^{2}\right)^{-1}=1$ does not belong to $C_{0}(\mathbf{R}, \mathbf{R})$.

\section{Isomorphisms of convolution algebras}

For a locally compact Hausdorff topological group $G$, let us regard $C_{c}(G, \mathbf{K})$ as an algebra under the convolution product

$$
(f * g)(x)=\int_{G} f(y) g\left(y^{-1} x\right) d y
$$

integration being with respect to a fixed left Haar measure denoted $d y$. We prepare the theorem of this section with two lemmas.

Lemma 4.1. Let $G_{1}$ and $G_{2}$ be locally compact Hausdorff topological groups, $T: C_{c}\left(G_{1}\right) \rightarrow C_{c}\left(G_{2}\right)$ a convolution algebra isomorphism and $\alpha$ : $G_{2} \rightarrow G_{1}$ a bijection such that, for any $f \in C_{c}\left(G_{1}\right), T f(y)=0$ is equivalent to $f(\alpha(y))=0$. Then $\alpha$ is a group isomorphism.

Proof. Suppose that $\alpha(x y) \neq \lambda(x) x(y)$ for some $x, y \in G_{2}$. Then there exist neighbourhoods $U \ni \alpha(x)$ and $V_{\ni} \wedge(y)$ such that $\alpha(x y) \notin U V$. Since $\alpha$ is continuous by an argument used at the beginning of the proof of Theorem 3.1, $\alpha^{-1}(U)$ is a neighbourhood of $x$ and $\alpha^{-1}(V)$ one of $y$. There exist non-negative real functions $f, g \in C_{c}\left(G_{2}\right)$ with $f(x)>0$, $g(y)>0$ such that $f$ vanishes outside $x^{-1}(U)$ and $g$ outside $\alpha^{-1}(V)$. By hypothesis, $T^{-1} f(t)=0$ for $t \in G_{1} \backslash U$ and $T^{-1} g(t)=0$ for $t \in G_{1} \backslash V$. Therefore, $\left(T^{-1} f\right) *\left(T^{-1} g\right)(t)=\int_{G_{1}} T^{-1} f(z) T^{-1} g\left(z^{-1} t\right) d z=0$ for all $t \in G_{1} \backslash U V$ the integrand being zero both in $U$ and $G_{1} \backslash U$. In particular,

$$
\left(T^{-1} f\right) *\left(T^{-1} g\right)(\alpha(x y))=0 .
$$


On the other hand, by continuity we can find a symmetric neighbourhood $W$ of the neutral element $e \in G_{2}$ such that $f(t)>\delta$ for $t \in x W, g(t)>\delta$ for $t \in W y$, where $\delta$ is a positive constant. Thus

$$
(f * g)(x y)=\int_{G_{2}} f(z) g\left(z^{-1} x y\right) d z==\int_{G_{2}} f(x z) g\left(z^{-1} y\right) d z \geq \int_{W} \delta^{2} d z>0 .
$$

The hypothesis then implies

$$
\left(T^{-1} f\right) *\left(T^{-1} g\right)(\alpha(x y))=T^{-1}(f * g)(\alpha(x y)) \neq 0,
$$

and this contradiction with (1) proves the lemma.

Lemma 4.2. Suppose $h$ is a continuous $\mathbf{K}$-valued function on the locally compact Hausdorff topological group $G$. The mapping $T: C_{c}(G, \mathbf{K})$ $\rightarrow C_{c}(G, \mathbf{K}), T f(x)=h(x) f(x)$ for $x \in G$, is a convolution algebra homomorphism if and only if $h(x y)=h(x) h(y)$ for all $x, y \in G$.

Proof. If $h$ is multiplicative, a straightforward calculation shows that $T$ preserves convolution. To prove the converse, choose for each compact neighbourhood $U$ of the neutral element $e$ a continuous non-negative real function $f_{U}$ such that $f_{U}$ vanishes outside $U$ and $\int_{G} f_{U}(x) d x=1$. We denote $f^{u}(t)=f\left(u^{-1} t\right)$ for a function $f \in C_{c}(G)$ and define $g_{U}^{(x, y)}=$ $f_{U}^{x} * f_{U}^{y}$ for each couple $(x, y)$ of elements of $G$. Then

$$
\int_{G} g_{U}^{(x, y)}(t) d t=1
$$

$g_{U}^{(x, y)}$ is non-negative and vanishes outside $V=x U y U$, and $V$ is eventually contained in any neighbourhood of $x y$, when the set $\mathcal{U}$ of the compact neighbourhoods of $e$ is given the natural order opposite to inclusion. It follows easily that

$$
h(x y)=\lim _{U} \int_{G} h(t) g_{U}^{(x, y)}(t) d t .
$$

Similarly,

$$
h(u)=\lim _{U} \int_{G} h(t) f_{U}^{u}(t) d t, u \in G
$$

Therefore, if $T$ preserves convolution, Fubini's theorem yields

$$
h(x y)=\lim _{U} \int_{G} h(t) g_{U}^{(x, y)}(t) d t=\lim _{U} \int_{G} T\left(f_{U}^{x} * f_{U}^{y}\right)(t) d t=
$$


$=\lim _{U} \int_{G}\left(T f_{U}^{x}\right) *\left(T f_{U}^{y}\right)(t) d t=\lim _{U} \int_{G} \int_{G} h(z)_{U}^{x}(z) h\left(z^{-1} t\right) f_{U}^{y}\left(z^{-1} t\right) d z d t=$

$=\lim _{U} \int_{G} h(z) f_{U}^{x}(z) d z \cdot \lim _{U} \int_{G} h(t) f_{U}^{y}(t) d t=h(x) h(y)$.

Theorem 4.1. Let $G_{1}$ and $G_{2}$ be locally compact Hausdorff spaces and $T: C_{c}\left(G_{1}, \mathbf{K}\right) \rightarrow C_{c}\left(G_{2}, \mathbf{K}\right)$ a bipositive (resp. isometric) vector space isomorphism Then there exist a homeomorphism $\alpha: G_{2} \rightarrow G_{1}$ and a continuous everywhere positive function $h$ on $G_{2}$ (resp. a continuous function $h: G_{2} \rightarrow \mathbf{K}$ with $\left.|h(y)|=1, y \in G_{2}\right)$ such that

$$
T f(y)=h(y) f(\alpha(y)), f \in C_{c}\left(G_{1}, \mathbf{K}\right), y \in G_{2} .
$$

Suppose, in addition, that $G_{1}$ and $G_{2}$ are topological groups. Then $T$ is a convolution algebra isomorphism if and only if $\alpha$ is a group isomorphism and $h(x y)=h(x) h(y)$ for all $x, y \in G_{2}$.

Proof. The first assertion is contained in Theorem 3.1, and the second is an immediate consequence of the above lemmas.

University of Helsinki

Helsinki, Finland 


\section{References}

[1] Dixuier, J.: Les $C^{*}$-algèbres et leurs représentations. - Cahiers scientifiques, Fas. XXIX, Gauthier-Villars, Paris (1961).

[2] Edwards, R. E.: Bipositive and isometric isomorphisms of some convolution algebras. - Canad. J. Math. 17, 839-\$46 (1965).

[3] Kaplansky, I.: Lattices of continuous functions I. - Bull. Amer. Math. Soc. $53,617-623$ (1947).

[4] -》- Lattices of continuous functions II. - Amer. J. Math. 70, 626-634 (1948).

[5] Кӧтне, G.: Topological vector spaces I. - Springer, Berlin-Heidelberg-New York (1969).

[6] Wendel, J. G.: On isometric isomorphisms of group algebras. - Pacific J. Math. $1,305-311$ (1951).

[7] Wood, G. V.: A note on isomorphisms of group algebras. - Proc. Amer. Math. Soc. $25,771-775(1970)$. 